\title{
$\lambda_{p c}$-open sets and $\lambda_{p c}$-separation axioms in topological spaces
}

\author{
Alias B. Khalaf *, Alan M. Omar \\ University of Duhok, Department of Mathematics \\ *Corresponding author E-mail: aliasbkhalaf@gmail.com
}

Copyright (C)2014 Alias B. Khalaf and Alan M. Omar. This is an open access article distributed under the Creative Commons Attribution License, which permits unrestricted use, distribution, and reproduction in any medium, provided the original work is properly cited.

\begin{abstract}
The aim of this paper is to introduce a new class of sets called $\lambda_{p c^{-}}$open sets and to investigate some of their relationships and properties. Further, by using this set, the notion of $\lambda_{p c}-T_{\mathrm{i}}$ spaces $(\mathrm{i}=0,1 / 2,1,2)$ and $\lambda_{p c}-R_{j}$ spaces $(\mathrm{j}=0,1)$ are introduced and some of their properties are investigated.

Keywords: s-operation; $\lambda_{p c}$-Open Set; $\lambda_{p c}-T_{i}, i=0,1,2 ; \lambda_{p c}-R_{j}, j=0,1$.
\end{abstract}

\section{Introduction}

The study of pre-open sets and pre-continuity in topological spaces was initiated by Mashhour, El-Monsef and El-Deeb [7]. Analogous to generalized closed sets which was introduced by Levine [8], Maki, Umehara and Nori [2], introduced the concept of pre-generalized closed sets in topological spaces. Kasahara [6], defined the concept of an operation on topological spaces and introduced the concept of closed graphs of a function. Ahmad and Hussain [1], continued studying the properties of operations on topological spaces introduced by Kasahara [6]. Ogata [10], introduced the concept of $\gamma-T_{\mathrm{i}}(\mathrm{i}=0,1 / 2,1,2)$ and characterized $\gamma$ - $T_{\mathrm{i}}$ by the notion of $\gamma$ - closed sets or $\gamma$-open sets. Chattopadhyay [9] defined other new types of separation axioms and Caldas, Jafari and Nori [5], defined pre- $R_{1}$, and pre- $R_{0}$ spaces.

In this paper, we introduce and study a new class of pre-open sets called $\lambda_{p c}$-open sets in topological spaces. By using the notion of $\lambda_{p c}$-closed and $\lambda_{p c}$-open sets, we introduce the concept of $\lambda_{p c}-T_{i}(i=0,1 / 2,1,2)$ and $\lambda_{p c}-R_{j}$ $(j=0,1)$ spaces. several properties and characterizations of these spaces are obtained.

\section{Preliminaries}

Throughout, $X$ denote a topological space with out any separation axiom. Let $A$ be a subset of $X$, the closure (interior) of $A$ are denoted by $C l(A)(\operatorname{Int}(A))$ respectively. A subset $A$ of a topological space $(X, \tau)$ is said to be pre-open [7] if $A \subseteq \operatorname{Int}(C l(A))$. The complement of a pre-open set is said to be pre-closed [7]. The family of all pre-open (resp. pre-closed) sets in a topological space $(X, \tau)$ is denoted by $P O(X, \tau)$ or $P O(X)(\operatorname{resp} . P C(X, \tau)$ or $P C(X))$. 
Definition 2.1 [4] Let $(X, \tau)$ be a topological space and let $A$ be a subset of $X$ then:

1. The pre-interior of $A(p \operatorname{Int}(A))$ is the union of all pre-open sets of $X$ contained in $A$.

2. A point $x \in X$ is said to be a pre-limit point of $A$ if every pre-open set containing $x$ contains a point of $A$ different from $x$, and the set of all pre-limit points of $A$ is called the pre-derived set of $A$ denoted by $p d(A)$.

3. The intersection of all pre-closed sets of $X$ containing $A$ is called the pre-closure of $A$ and is denoted by $p C l(A)$.

Definition 2.2 [2] A subset $A$ of a space $(X, \tau)$ is called a pre-generalized closed set (pg-closed), if $A \subseteq U$ and $U$ is pre-open implies that $p C l(A) \subseteq U$.

Definition 2.3 A topological space $(X, \tau)$ is said to be:

1. pre- $T_{0}[9]$ if for any distinct pair of points in $X$, there is an pre-open set containing one of the points but not the other.

2. pre- $T_{1}[9]$ if for any distinct pair of points $x$ and $y$ in $X$, there is a pre-open $U$ in $X$ containing $x$ but not $y$ and a pre-open set $V$ in $X$ containing $y$ but not $x$.

3. pre- $T_{2}$ [9] if for any distinct pair of points $x$ and $y$ in $X$, there exist pre-open sets $U$ and $V$ in $X$ containing $x$ and $y$, respectively, such that $U \cap V=\phi$.

4. pre-T $T_{1 / 2}$ [2] if every pg-closed set is pre-closed.

5. pre- $R_{0}[5]$ if for each $O \in P O(X)$ and $x \in O, p C l(\{x\}) \subseteq O$.

6. pre- $R_{1}[5]$ if for each pair $x, y \in X$ such that $p C l(\{x\}) \neq p C l(\{y\})$, there exist disjoint pre-open sets $U$ and $V$ such that $p C l(\{x\}) \subseteq U$ and $p C l(\{y\}) \subseteq V$.

Definition 2.4 [10] Let $(X, \tau)$ be any topological space. A mapping $\lambda: \tau \rightarrow P(X),(P(X)$ stands for all subsets of $X)$, is called an operation on $\tau$ if $V \subseteq \lambda(V)$ for each non-empty open set $V$ and $\lambda(\phi)=\phi$.

Definition 2.5 [3] Let $(X, \tau)$ be a topological space and let $A$ be a subset of $X$ then:

1. The $\lambda$-interior of $A\left({ }_{\lambda} \operatorname{Int}(A)\right)$ is the union of all $\lambda$-open sets of $X$ contained in $A$.

2. A point $x \in X$ is said to be a $\lambda$-limit point of $A$ if every $\lambda$-open set containing $x$ contains a point of $A$ different from $x$, and the set of all $\lambda$-limit points of $A$ is called the $\lambda$-derived set of $A$ denoted by ${ }_{\lambda} d(A)$.

3. The intersection of all $\lambda$-closed sets of $X$ containing $A$ is called the $\lambda$-closure of $A$ and is denoted by ${ }_{\lambda} C l(A)$.

\section{3. $\lambda_{p c}$-Open sets}

In this section, we introduce a new class of pre-open sets called $\lambda_{p c}$-open sets. Further, the notion of $\lambda_{p c}$-derived set, $\lambda_{p c}$-closure and $\lambda_{p c}$-interior are introduced and their properties are discussed.

Definition 3.1 A mapping $\lambda: P O(X) \rightarrow P(X)$ is called an p-operation on $P O(X)$ if $V \subseteq \lambda(V)$ for each non-empty pre-open set $V$ and $\lambda(\phi)=\phi$.

If $\lambda: P O(X) \rightarrow P(X)$ is any p-operation, then it is clear that $\lambda(X)=X$.

Definition 3.2 Let $(X, \tau)$ be a topological space and $\lambda: P O(X) \rightarrow P(X)$ be an p-operation defined on $P O(X)$, then a subset $A$ of $X$ is $\lambda$-open set if for each $x \in A$ there exists a pre-open set $U$ such that $x \in U$ and $\lambda(U) \subseteq A$.

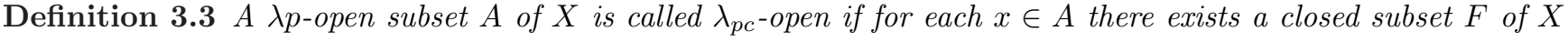
such that $x \in F \subseteq A$.

The complement of a $\lambda_{p c}$-open set is said to be $\lambda_{p c}$-closed. The family of all $\lambda_{p c}$-open ( resp., $\lambda_{p c}$-closed ) subsets of a topological space $(X, \tau)$ is denoted by $P O_{\lambda p c}(X, \tau)$ or $P O_{\lambda \mathrm{pc}}(X)\left(\operatorname{resp} ., P C_{\lambda p c}(X, \tau)\right.$ or $\left.P C_{\lambda p c}(X)\right)$

Proposition 3.4 For any topological space $(X, \tau)$, we have $P O_{\lambda p c}(X) \subseteq P O_{\lambda}(X) \subseteq P O(X)$. 
Proof. Obvious

The following examples show that the equality in the above proposition may not be true in general.

Example 3.5 Let $X=\{a, b, c\}$, and $\tau=\{\phi,\{a\},\{a, b\}, X\}$. We define an p-operation $\lambda: P O(X) \rightarrow P(X)$ as $\lambda(A)=A$ if $A=\{a, c\}$ or $A$ is empty and $\lambda(A)=X$ otherwise. Here, we have $\{a, c\}$ is $\lambda$-open set but it is not $\lambda_{\text {pc-open. }}$

The following examples shows that $\tau$ is incomparable with $\lambda_{p c} O(X)$.

Example 3.6 Let $X=\{a, b, c\}$, and $\tau=\{\phi,\{a\},\{a, b\}, X\}$. We define an p-operation $\lambda: P O(X) \rightarrow P(X)$ as $\lambda(A)=A$ if $A \neq\{a\}$ or $\{b\}$ and $\lambda(A)=\{a, b\}$ if $A=\{a\}$ or $\{b\}$. Now, we have $\{a\}$ is open set but not $\lambda_{p c}{ }^{-o p e n}$.

Example 3.7 Let $N$ be a set of natural numbers. In a topological space $(N, \tau)$ with cofinite topology. We define

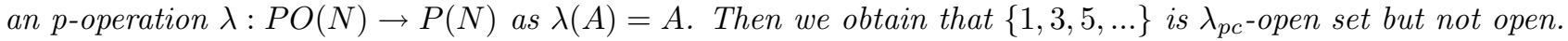

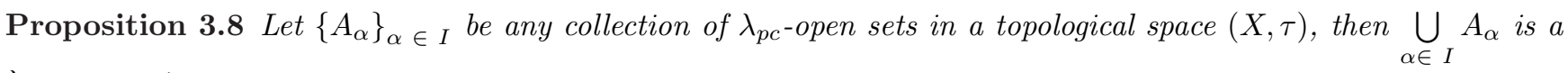
$\lambda_{p c^{-} \text {open set. }}$

Proof. Since $A_{\alpha}$ is $\lambda_{p c}$-open set for all $\alpha \in I$, then $A_{\alpha}$ is a $\lambda p$-open set for all $\alpha \in I$. This implies that there exists a pre-open set $U$ such that $\lambda(U) \subseteq A_{\alpha_{0}} \subseteq \bigcup_{\alpha \in I} A_{\alpha}$. Therefore, $\bigcup_{\alpha \in I} A_{\alpha}$ is a $\lambda p$-open subset of $(X, \tau)$. Let $x \in \bigcup_{\alpha \in I} A_{\alpha}$, then there exists an $\alpha_{0} \in I$ such that $x \in A_{\alpha 0}$. Since $A_{\alpha}$ is a $\lambda_{p c}$-open set for all $\alpha \in I$, then there exists a closed set $F$ such that $x \in F \subseteq A_{\alpha 0}$ but $A_{\alpha 0} \subseteq \bigcup_{\alpha \in I} A_{\alpha}$, then $x \in F \subseteq \bigcup_{\alpha \in I} A_{\alpha}$. Hence, $\bigcup_{\alpha \in I} A_{\alpha}$ is $\lambda_{p c}$-open.

The following example shows that the intersection of two $\lambda_{p c}$-open sets need not be $\lambda_{p c}$-open.

Example 3.9 Let $X=\{a, b, c\}$ and $\tau=P(X)$. We define an p-operation $\lambda: P O(X) \rightarrow P(X)$ as $\lambda(A)=A$ if $A$ is

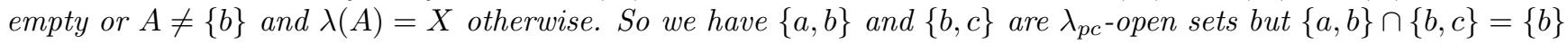

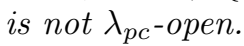

Proposition 3.10 The set $A$ is $\lambda_{p c}$-open in the space $(X, \tau)$ if and only if for each $x \in A$ there exists a $\lambda_{p c}$-open set $B$ such that $x \in B \subseteq A$.

Proof. Suppose that $A$ is $\lambda_{p c}$-open in $(X, \tau)$. Then for each $x \in A$ we put $B=A$ is a $\lambda_{p c}$-open set such that $x \in B \subseteq A$.

Conversely, Suppose that for each $x \in A$ there exists a $\lambda_{p c}$-open set $B_{x}$ such that $x \in B_{x} \subseteq A$. Thus $A=\bigcup B_{x}$, where $B_{x} \in P O_{\lambda p c}(X)$ for each $x$. Therefore, by Proposition 3.8, $A$ is $\lambda_{p c^{-o p e n}}$.

Definition 3.11 Let $(X, \tau)$ be a topological space. An p-operation $\lambda$ is said to be p-regular if for every pre-open sets $U$ and $V$ containing $x \in X$, there exists a pre-open set $W$ containing $x$ such that $\lambda(W) \subseteq \lambda(U) \cap \lambda(V)$.

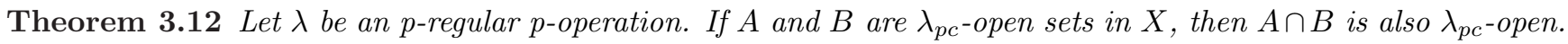

Proof. Let $x \in A \cap B$, then $x \in A$ and $x \in B$. Since $A$ and $B$ are $\lambda p$-open sets, so there exist pre-open sets $U$ and $V$ such that $x \in U$ and $\lambda(U) \subseteq A, x \in V$ and $\lambda(V) \subseteq B$. Since $\lambda$ is p-regular, this implies that there exists a pre-open set $W$ of $x$ such that $\lambda(W) \subseteq \lambda(U) \cap \lambda(V) \subseteq A \cap B$. Therefore, $A \cap B$ is $\lambda p$-open set. Again for each

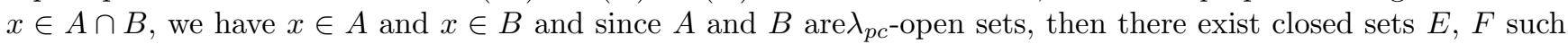
that $x \in E \subseteq A$ and $x \in F \subseteq B$. Therefore, $x \in E \cap F \subseteq A \cap B$. Since $E \cap F$ is closed, so by Definition 3.2, we obtain that $A \cap B$ is $\lambda_{p c^{-o p e n}}$.

Definition 3.13 Let $(X, \tau)$ be a topological space and let $A$ be subset of $X$, then a point $x \in X$ is called a $\lambda_{\text {pc-limit }}$ point of $A$ if every $\lambda_{p c^{-}}$open set containing $x$ contains a point of $A$ different from $x$.

The set of all $\lambda_{p c}$-limit points of $A$ is called the $\lambda_{p c}$-derived set of $A$ denoted by $\lambda_{p c} d(A)$.

Definition 3.14 Let $A$ be subset of the space $(X, \tau)$, then the $\lambda_{p c}$-closure of $A\left(\lambda_{p c} C l(A)\right)$ is the intersection of all $\lambda_{p c}$-closed sets containing $A$.

Here we introduce some properties of $\lambda_{p c}$-closure of the sets.

Proposition 3.15 For subsets $A, B$ of a topological space $(X, \tau)$, the following statements are true. 
1. $A \subseteq \lambda_{p c} C l(A)$.

2. $\lambda_{p c} C l(A)$ is $\lambda_{p c}$-closed set in $X$.

3. $\lambda_{p c} C l(A)$ is smallest $\lambda_{p c}$-closed set which contain $A$.

4. $A$ is $\lambda_{p c}$-closed set if and only if $A=\lambda_{p c} C l(A)$.

5. $\lambda_{p c} C l(\phi)=\phi$ and $\lambda_{p c} C l(X)=X$.

6. If $A \subseteq B$. Then $\lambda_{p c} C l(A) \subseteq \lambda_{p c} C l(B)$.

7. $\lambda_{p c} C l(A) \cup \lambda_{p c} C l(B) \subseteq \lambda_{p c} C l(A \cup B)$.

8. $\lambda_{p c} C l(A \cap B) \subseteq \lambda_{p c} C l(A) \cap \lambda_{p c} C l(B)$.

Proof. Obvious.

In general the equalities (7) and (8) of the above proposition is not true, as it is shown in the following examples:

Example 3.16 Let $X=\{a, b, c\}$, and $\tau=P(X)$. We define an p-operation $\lambda: P O(X) \rightarrow P(X)$ as $\lambda(A)=A$ if $A=\phi, A=\{a, b\}$ or $\{b, c\}$ and $\lambda(A)=X$ otherwise. Now, if $A=\{b\}$ and $B=\{c\}$, then $\lambda_{p c} C l(A)=\{b\}$ and $\lambda_{p c} C l(B)=\{c\}$, but $\lambda_{p c} C l(A \cup B)=X$, where $A \cup B=\{b, c\} . \lambda_{p c} C l(A \cup B) \neq \lambda_{p c} C l(A) \cup \lambda_{p c} C l(B)$.

Example 3.17 Let $X=\{a, b, c\}, \tau=\{\phi,\{a\},\{c\},\{a, c\}, X\}$. We define an p-operation $\lambda: P O(X) \rightarrow P(X)$ as $\lambda(A)=A$ if $A=\phi$ or $c \in A$ and $\lambda(A)=C l(A)$ otherwise. Now, if $A=\{a\}$ and $B=\{b\}$ then $\lambda_{p c} C l(A)=\{a, b\}$ and $\lambda_{p c} C l(B)=\{b\}$, but $\lambda_{p c} C l(A \cap B)=\phi$, where $A \cap B=\phi$. Hence $\lambda_{p c} C l(A \cap B) \neq \lambda_{p c} C l(A) \cap \lambda_{p c} C l(B)$.

Proposition 3.18 Let $A$ be any subset of a space $X$, then $\lambda_{p c} C l(A)=A \cup \lambda_{p c} d(A)$.

Proof. Obvious.

Proposition 3.19 If $A$ is a subset of $(X, \tau)$, then $x \in \lambda_{p c} C l(A)$ if and only if $V \cap A \neq \phi$ for every $\lambda_{p c^{-o p e n ~ s e t ~} V}$ containing $x$.

Proof. Let $x \in \lambda_{p c} C l(A)$ and suppose that $V \cap A=\phi$ for some $\lambda_{p c}$-open set $V$ which contains $x$. This implies that $X \backslash V$ is $\lambda_{p c}$-closed and $A \subseteq(X \backslash V)$, so $\lambda_{p c} C l(A) \subseteq(X \backslash V)$. But this implies that $x \in(X \backslash V)$ which is contradiction. Therefore, $V \cap A \neq \phi$.

Conversely, Let $A \subseteq X$ and $x \in X$ such that for each $\lambda_{p c}$-open set $V$ containing $x, V \cap A \neq \phi$. If $x \notin \lambda_{p c} C l(A)$, then there is a $\lambda_{p c}$-closed set $S$ such that $A \subseteq S$ and $x \notin S$. Hence, $(X \backslash S)$ is a $\lambda_{p c}$-open set with $x \in(X \backslash S)$ and thus $(X \backslash S) \cap A \neq \phi$ which is a contradiction. Therefore, $x \in \lambda_{p c} C l(A)$.

Proposition 3.20 If $A$ is any subset of a topological space $(X, \tau)$, then ${ }_{p} C l(A) \subseteq \lambda_{p c} C l(A)$.

Proof. Obvious.

The following example shows that the equality in the above proposition is not true in general.

Example 3.21 Let $X=\{a, b, c\}$, and $\tau=\{\phi,\{a\},\{a, b\}, X\}$. We define an p-operation $\lambda: P O(X) \rightarrow P(X)$ as $\lambda(A)=A$ if $A=\phi$ or $A=\{a\}$ and $\lambda(A)=X$ otherwise. Now, if $A=\{c\}$, then ${ }_{p} C l(A)=\{c\}$ and $\lambda_{p c} C l(A)=X$.

Definition 3.22 Let $(X, \tau)$ be a topological space and let $A$ be subset of $X$, then the $\lambda_{p c}$-interior of $A\left(\lambda_{p c} \operatorname{Int}(A)\right)$ is the union of all $\lambda_{p c^{-}}$open sets of $X$ contained in $A$.

Proposition 3.23 For subsets $A, B$ of a space $X$, the following statements hold.

1. $\lambda_{p c} \operatorname{Int}(A)$ is the union of all $\lambda_{p c}$-open sets which are contained in $A$.

2. $\lambda_{p c} \operatorname{Int}(A)$ is a $\lambda_{p c}$-open set in $X$.

3. $\lambda_{p c} \operatorname{Int}(A) \subseteq A$.

4. $\lambda_{p c} \operatorname{Int}(A)$ is the largest $\lambda_{p c}$-open set contained in $A$.

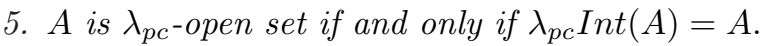


6. $\lambda_{p c} \operatorname{Int}\left(\lambda_{p c} \operatorname{Int}(A)\right)=\lambda_{p c} \operatorname{Int}(A)$.

7. If $A \subseteq B$, then $\lambda_{p c} \operatorname{Int}(A) \subseteq \lambda_{p c} \operatorname{Int}(B)$.

8. $\lambda_{p c} \operatorname{Int}(\phi)=\phi$ and $\lambda_{p c} \operatorname{Int}(X)=X$.

9. $\lambda_{p c} \operatorname{Int}(A) \cup \lambda_{p c} \operatorname{Int}(B) \subseteq \lambda_{p c} \operatorname{Int}(A \cup B)$.

10. $\lambda_{p c} \operatorname{Int}(A \cap B) \subseteq \lambda_{p c} \operatorname{Int}(A) \cap \lambda_{p c} \operatorname{Int}(B)$.

Proof. Obvious.

In general the equalities of (9) and (10) of the above proposition is not true, as it is shown in the following examples:

Example 3.24 Let $X=\{a, b, c\}$, and $\tau=\{\phi,\{a\},\{b\},\{a, b\},\{a, c\}, X\}$. We define an p-operation $\lambda: P O(X) \rightarrow$ $P(X)$ as $\lambda(A)=A$ if $b \in A$ and $\lambda(A)=C l(A)$ if $b \notin A$. Now, let $A=\{a\}$ and $B=\{c\}$, then $\lambda_{p c} \operatorname{Int}(A)=\phi$, and $\lambda_{p c} \operatorname{Int}(B)=\phi$, but $\lambda_{p c} \operatorname{Int}(A \cup B)=\{a, c\}$. Thus $\lambda_{p c} \operatorname{Int}(A \cup B) \neq \lambda_{p c} \operatorname{Int}(A) \cup \lambda_{c} \operatorname{Int}(B)$.

Example 3.25 Let $X=\{a, b, c\}$, and $\tau=P(X)$. We define an p-operation $\lambda: P O(X) \rightarrow P(X)$ as $\lambda(A)=A$ if $A=\phi, A=\{c\},\{a, b\}$ or $\{a, c\}$ and $\lambda(A)=X$ otherwise. Now, if $A=\{a, b\}$ and $B=\{a, c\}$, then $\lambda_{p c} \operatorname{Int}(A)=$ $\{a, b\}$ and $\lambda_{p c} \operatorname{Int}(B)=\{a, c\}$, but $\lambda_{p c} \operatorname{Int}(A \cap B)=\phi$. Hence, $\lambda_{p c} \operatorname{Int}(A \cap B) \neq \lambda_{p c} \operatorname{Int}(A) \cap \lambda_{p c} \operatorname{Int}(B)$.

Proposition 3.26 if $A$ is a subset of a space $X$, then $\lambda_{p c} \operatorname{Int}(A)=A \backslash \lambda_{p c} d(X \backslash A)$.

Proof. Obvious.

Proposition 3.27 If $A$ is any subset of a space $X$, then the following statements are true:

1. $X \backslash \lambda_{p c} \operatorname{Int}(A)=\lambda_{p c} C l(X \backslash A)$.

2. $\lambda_{p c} C l(A)=X \backslash \lambda_{p c} \operatorname{Int}(X \backslash A)$.

3. $X \backslash \lambda_{p c} C l(A)=\lambda_{p c} \operatorname{Int}(X \backslash A)$.

4. $\lambda_{p c} \operatorname{Int}(A)=X \backslash \lambda_{p c} C l(X \backslash A)$.

Proof. Obvious.

Proposition 3.28 If $A$ is a subset of a topological space $(X, \tau)$, then $\lambda_{p c} \operatorname{Int}(A) \subseteq{ }_{p} \operatorname{Int}(A)$.

Proof. Obvious.

The equality in the above proposition need not be true in general, as shown by the following example:

Example 3.29 Let $X=\{a, b, c\}$, and $\tau=\{\phi,\{a\},\{b\},\{a, b\}, X\}$. We define an p-operation $\lambda: P O(X) \rightarrow P(X)$ as $\lambda(A)=A$ if $A$ is empty or $A=\{b\}$ and $\lambda(A)=X$ otherwise. Now, if $A=\{a, b\}$, then $\lambda_{p c} \operatorname{Int}(A)=\phi$ and ${ }_{p} \operatorname{Int}(A)=\{a, b\}$.

Theorem 3.30 Let $A, B$ be subsets of $X$. If the p-operation $\lambda: P O(X) \rightarrow P(X)$ is s-regular, then we have:

1. $\lambda_{p c} C l(A \cup B)=\lambda_{p c} C l(A) \cup \lambda_{p c} C l(B)$.

2. $\lambda_{p c} \operatorname{Int}(A \cap B)=\lambda_{p c} \operatorname{Int}(A) \cap \lambda_{p c} \operatorname{Int}(B)$.

Proof. Obvious. 


\section{4. $\quad \lambda_{p c}$-Separation axioms}

In this section, we define new types of separation axioms called $\lambda_{p c}-T_{i}(i=0,1 / 2,1,2)$ and $\lambda_{p c}-R_{j}(j=0,1)$ by using the notion of $\lambda_{p c}$-open and $\lambda_{p c}$-closed sets. First, we begin with the following definition.

Definition 4.1 A subset $A$ of $(X, \tau)$ is said to be generalized $\lambda_{p c}$-closed (briefly $g$ - $\lambda_{p c}$-closed) if $\lambda_{p c} C l(A) \subseteq U$,

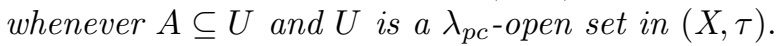

We say that a subset $B$ of $X$ is generalized $\lambda_{p c}$-open (briefly. $g-\lambda_{p c}$-open) if its complement $X \backslash B$ is generalized $\lambda_{p c^{-c}}$ closed in $(X, \tau)$.

Theorem 4.2 If a subset $A$ of $X$ is $g-\lambda_{p c}$-closed and $A \subseteq B \subseteq \lambda_{p c} C l(A)$, then $B$ is a $g$ - $\lambda_{p c}$-closed set in $X$.

Proof. Let $A$ be $g$ - $\lambda_{p c}$-closed set such that $A \subseteq B \subseteq \lambda_{p c} C l(A)$. Let $U$ be a $\lambda_{p c}$-open set of $X$ such that $B \subseteq U$. Since $A$ is $g$ - $\lambda_{p c}$-closed, we have $\lambda_{p c} \mathrm{Cl}(\mathrm{A}) \subseteq U$. Now $\lambda_{p c} C l(A) \subseteq \lambda_{p c} C l(B) \subseteq \lambda_{p c} C l\left(\lambda_{p c} C l(A)\right)=\lambda_{p c} C l(A) \subseteq U$. This implies that $\lambda_{p c} C l(B) \subseteq U$, where $U$ is $\lambda_{p c}$-open. Therefore, $B$ is a $g$ - $\lambda_{p c}$-closed set in $X$.

In the following example, we have two $g$ - $\lambda_{p c}$-closed sets $A$ and $B$ such that $A \subseteq B$ but $B \not \subset \lambda_{p c} C l(A)$.

Example 4.3 Let $X=\{a, b, c\}$, and $\tau=\{\phi,\{a\},\{c\},\{a, c\},\{b, c\}, X\}$. Let $\lambda: P O(X) \rightarrow P(X)$ be identity poperation. If $A=\{a\}$ and $B=\{a, c\}$, then $A$ and $B$ are $g-\lambda_{p c}$-closed sets in $(X, \tau)$. But $A \subseteq B \not \subset \lambda_{p c} C l(A)$.

Theorem 4.4 Let $\lambda: P O(X) \rightarrow P(X)$ be an p-operation, then for each singleton set $\{x\}$ is $\lambda_{p c}$-closed or $X \backslash\{x\}$ is $g$ - $\lambda_{p c}$-closed in $(X, \tau)$.

Proof. Suppose that $\{x\}$ is not $\lambda_{p c}$-closed, then $X \backslash\{x\}$ is not $\lambda_{p c}$-open. Let $U$ be any $\lambda_{p c}$-open set such that $X \backslash\{x\} \subseteq U$, then $U=X$. Therefore $\lambda_{p c} C l(X \backslash\{x\}) \subseteq U$. Hence $X \backslash\{x\}$ is $g$ - $\lambda_{p c}$-closed.

Proposition 4.5 A subset $A$ of $(X, \tau)$ is $g-\lambda_{p c}$-closed if and only if $\lambda_{p c} C l(\{x\}) \cap A \neq \phi$, for every $x \in \lambda_{p c} C l(A)$.

Proof. Let $U$ be a $\lambda_{p c}$-open set such that $A \subseteq U$ and let $x \in \lambda_{p c} C l(A)$. By assumption, there exists a $z \in \lambda_{p c} C l(\{x\})$ and $z \in A \subseteq U$. It follows From Proposition 3.19, that $U \cap\{x\} \neq \phi$, hence $x \in U$, implies $\lambda_{p c} C l(A) \subseteq U$. Therefore $A$ is $g$ - $\lambda_{p c}$-closed.

Conversely, suppose that $x \in \lambda_{p c} C l(A)$ such that $\lambda_{p c} C l(\{x\}) \cap A=\phi$. Since $A \subseteq X \backslash \lambda_{p c} C l(\{x\})$ and $A$ is $g-\lambda_{p c^{-}}$ closed implies that $\lambda_{p c} C l(A) \subseteq X \backslash \lambda_{p c} C l(\{x\})$ holds, and hence $x \notin \lambda_{p c} C l(A)$, which is contradiction. Therefore $\lambda_{p c} C l(\{x\}) \cap A \neq \phi$.

Theorem 4.6 If a subset $A$ of $X$ is $g$ - $\lambda_{p c}$-closed set in $X$, then $\lambda_{p c} C l(A) \backslash A$ does not contain any non empty $\lambda_{p c}$-closed set in $X$.

Proof. Let $A$ be a $g$ - $\lambda_{p c}$-closed set in $X$. Let $F$ be a $\lambda_{p c}$-closed set such that $F \subseteq \lambda_{p c} C l(A) \backslash A$ and $F \neq \phi$. Then $F \subseteq X \backslash A$ which implies that $A \subseteq X \backslash F$. Since $A$ is $g$ - $\lambda_{p c}$-closed and $X \backslash F$ is a $\lambda_{p c}$-open set, therefore $\lambda_{p c} C l(A) \subseteq X \backslash F$, so $F \subseteq X \backslash \lambda_{p c} C l(A)$. Hence $F \subseteq \lambda_{p c} C l(A) \cap X \backslash \lambda_{p c} C l(A)=\phi$. This shows that, $F=\phi$ which is a contradiction. Hence $\lambda_{p c} C l(A) \backslash A$ does not contains any non empty $\lambda_{p c}$-closed set in $X$.

Definition 4.7 Let $(X, \tau)$ be a topological space then $(X, \tau)$ is said to be:

1. a $\lambda_{p c}-T_{0}$ space if for each distinct points $x, y \in X$ there exists a $\lambda_{p c}$-open set $U$ such that $x \in U$ and $y \notin U$ or $y \in U$ and $x \notin U$.

2. a $\lambda_{p c}-T_{1 / 2}$ space if every $g-\lambda_{p c}$-closed set in $(X, \tau)$ is $\lambda_{p c}$-closed.

3. a $\lambda_{p c}-T_{1}$ space if for each distinct points $x, y \in X$, there exists a $\lambda_{p c}$-open set, containing and respectively such that $y \notin U$ and $x \notin V$.

4. a $\lambda_{p c}-T_{2}$ space if for each $x, y \in X$ there exists a $\lambda_{p c}$-open sets $U, V$ such that $x \in U$ and $y \in V$ and $U \cap V \neq \phi$.

Proposition 4.8 Each $\lambda_{p c}-T_{i}$ space is pre- $T_{i}(i=0,1 / 2,1,2)$.

Proof. Obvious.

The following example show that every pre- $T_{i}$ space need not be $\lambda_{p c}-T_{i}(i=0,1 / 2,1,2)$. 
Example 4.9 Let $X=\{a, b, c\}$, and $\tau=P(X)$. We define an $p$-operation $\lambda: P O(X) \rightarrow P(X)$ as $\lambda(A)=A$ if $A=\{a\}$ and $\lambda(A)=X$ otherwise. Then the space $X$ is a pre- $T_{0}$ but it is not $\lambda_{p c}-T_{0}$ space. Moreover a space is pre- $T_{i}$, for $i=0,1 / 2,1,2$.

Theorem 4.10 A space $X$ is $\lambda_{p c}-T_{0}$ if and only if for each distinct points $x$ and $y$ in $X$, either $x \notin \lambda_{p c} C l(\{y\})$ or $y \notin \lambda_{p c} C l(\{x\})$.

Proof. Let $x \neq y$ in a $\lambda_{p c}-T_{0}$ space $X$. Then there exists an $\lambda_{p c}$-open set $U$ containing one of them but not the other, without loss of generality, we assume that $U$ contains $x$ but not $y$. Then $U \cap\{y\}=\phi$, this implies that $x \notin \lambda_{p c} C l(\{y\})$.

Conversely, Let $x$ and $y$ be two distinct points of $X$, then by hypothesis, either $x \notin \lambda_{p c} C l(\{y\})$ or $y \notin \lambda_{p c} C l(\{x\})$. With out loss of generality, we assume that $y \notin \lambda_{p c} C l(\{x\})$. Then $X \backslash \lambda_{p c} C l(\{x\})$ is an $\lambda_{p c}$-open subset of $X$ containing $y$ but not $x$. Therefore, $X$ is $\lambda_{p c}-T_{0}$.

Theorem 4.11 Let $\lambda: P O(X) \rightarrow P(X)$ be an p-operation, then the following statements are equivalent:

1. $(X, \tau)$ is $\lambda_{p c}-T_{1 / 2}$.

2. Each singleton $\{x\}$ of $X$ is either $\lambda_{p c}$-closed or $\lambda_{p c}$-open.

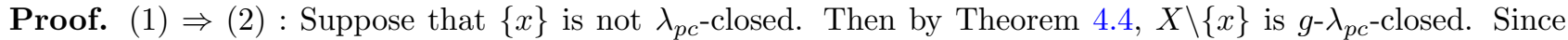
$(X, \tau)$ is $\lambda_{p c}-T_{1 / 2}$, then $X \backslash\{x\}$ is $\lambda_{p c}$-closed. Hence, $\{x\}$ is $\lambda_{p c}$-open.

$(2) \Rightarrow(1)$ : Let $A$ be any $g-\lambda_{p c}$-closed set in $(X, \tau)$ and $x \in \lambda_{p c} C l(A)$. By (2), we have $\{x\}$ is $\lambda_{p c}$-closed or $\lambda_{p c}$-open. If $\{x\}$ is $\lambda_{p c}$-closed and $x \notin A$ will imply $x \in \lambda_{p c} C l(A) \backslash A$ which is not true by Theorem 4.6, so $x \in A$. Therefore, $\lambda_{p c} C l(A)=A$, so $A$ is $\lambda_{p c^{-}}$closed. Therefore, $(X, \tau)$ is $\lambda_{p c^{-}} T_{1 / 2}$.

On the other hand, if $\{x\}$ is $\lambda_{p c}$-open, then as $x \in \lambda_{p c} C l(A)$,, we have $\{x\} \cap A \neq \phi$. Hence $x \in A$, so $A$ is $\lambda_{p c}$-closed.

Corollary 4.12 Each $\lambda_{p c}-T_{1 / 2}$ space is $\lambda_{p c}-T_{0}$ space.

Proof. Follows from Theorem 4.11 and Theorem 4.10.

Example 4.13 Let $X=\{a, b, c\}$ and $\tau=P(X)$. We define an p-operation $\lambda: P O(X) \rightarrow P(X)$ as $\lambda(A)=A$ if $A$ is empty, $A=\{a\}$ or $\{a, b\}$ and $\lambda(A)=X$ otherwise. Then $(X, \tau)$ is a $\lambda_{p c}-T_{0}$ space but not $\lambda_{p c}-T_{1 / 2}$ space because $\{a, b\}$ is $g-\lambda_{p c^{-}}$closed but not $\lambda_{p c^{-}}$closed.

Theorem 4.14 Each $\lambda_{p c}-T_{1}$ space is $\lambda_{p c}-T_{1 / 2}$ space.

Proof. Follows from Theorem 4.6.

Example 4.15 $X=\{a, b\}$, and $\tau=P(X)$. We define an p-operation $\lambda: P O(X) \rightarrow P(X)$ as $\lambda(A)=A$ if $A$ is empty and $\lambda(A)=X$ otherwise. Then $(X, \tau)$ is a $\lambda_{p c}-T_{1 / 2}$ space but not $\lambda_{p c}-T_{1}$ space.

Definition 4.16 A topological space $(X, \tau)$ is called a $\lambda_{p c}$-symmetric space if for $x$ and $y$ in $X, x \in \lambda_{p c} C l(\{y\})$ implies that $y \in \lambda_{p c} C l(\{x\})$.

Theorem 4.17 Let $(X, \tau)$ be a $\lambda_{p c}$-symmetric space, then the following are equivalent:

1. $(X, \tau)$ is $\lambda_{p c}-T_{0}$.

2. $(X, \tau)$ is $\lambda_{p c}-T_{1 / 2}$.

3. $(X, \tau)$ is $\lambda_{p c}-T_{1}$.

Proof. It is enough to prove only the necessity of $(1) \Leftrightarrow(2)$. Let $x \neq y$ and since $(X, \tau)$ is $\lambda_{p c}$ - $\mathrm{T}_{0}$, we may assume that $x \in U \subseteq X \backslash\{y\}$ for some $U \in P O_{\lambda p c}(X)$. Then $x \notin \lambda_{p c} C l(\{y\})$ and hence $y \notin \lambda_{p c} C l(\{x\})$. Therefore, there exists $V \in P O_{\lambda \mathrm{pc}}(X)$ such that $y \in V \subseteq X \backslash\{x\}$ and $(X, \tau)$ is a $\lambda_{p c}-T_{1}$ space.

Remark 4.18 From the definitions of $\lambda_{p c}-T_{i},(i=0,1 / 2,1,2)$ and previous results, we get the following diagram of implications:

$\lambda_{p c}-T_{2} \Rightarrow \lambda_{p c}-T_{1} \Rightarrow \lambda_{p c}-T_{1 / 2} \Rightarrow \lambda_{p c}-T_{0}$ 
Definition 4.19 Let $\lambda: P O(X) \rightarrow P(X)$ be an p-operation, a topological space $(X, \tau)$ is called $\lambda_{p c}-R_{0}$ if $U \in$ $P O_{\lambda p c}(X)$ and $x \in U$, then $\lambda_{p c} C l(\{x\}) \subseteq U$.

Theorem 4.20 For any topological space $X$ and any s-operation $\lambda$, the following are equivalent:

1. $X$ is $\lambda_{p c}-R_{0}$.

2. $F \in P C_{\lambda p c}(X)$ and $x \notin F$ implies $F \subseteq U$ and $x \notin U$ for some $U \in P O_{\lambda p c}(X)$.

3. $F \in P C_{\lambda p c}(X)$ and $x \notin F$ implies $F \cap \lambda_{p c} C l(\{x\})=\phi$.

4. For any two distinct points $x$, y of $X$, either $\lambda_{p c} C l(\{x\})=\lambda_{p c} C l(\{y\})$ or $\lambda_{p c} C l(\{x\}) \cap \lambda_{p c} C l(\{y\})=\phi$.

Proof. (1) $\Rightarrow(2): F \in P C_{\lambda \mathrm{pc}}(X)$ and $x \notin F$ implies $x \in X \backslash F \in P O_{\lambda \mathrm{pc}}(X)$ then $\lambda_{p c} C l(\{x\}) \subseteq X \backslash F$. By (1), if we put $U=X \backslash \lambda_{p c} C l(\{x\})$, then $x \notin U \in P O_{\lambda \mathrm{pc}}(X)$ and $F \subseteq U$.

$(2) \Rightarrow(3):$ if $F \in P C_{\lambda \mathrm{pc}}(X)$ and $x \notin F$, then there exists $U \in P O_{\lambda \mathrm{pc}}(X)$ such that $x \notin U$ and $F \subseteq U$. By (2), we have $U \cap \lambda_{p c} C l(\{x\})=\phi$, so $F \cap \lambda_{p c} C l(\{x\})=\phi$.

$(3) \Rightarrow(4)$ : Suppose that for any two distinct points $x, y$ of $X, \lambda_{p c} C l(\{x\}) \neq \lambda_{p c} C l(\{y\})$. Then suppose, without loss of generality, that there exists some $z \in \lambda_{p c} C l(\{x\})$ such that $z \notin \lambda_{p c} C l(\{y\})$. Thus there exists a $\lambda_{p c}$-open set $V$ such that $z \in V$ and $y \notin V$ but $x \in V$. Thus $x \notin \lambda_{p c} C l(\{y\})$. Hence by (3), $\lambda_{p c} C l(\{x\}) \cap \lambda_{p c} C l(\{y\})=\phi$.

$(4) \Rightarrow(1)$ : Let $U \in P O_{\lambda \mathrm{pc}}(X)$ and $x \in U$. Then for each $y \notin U, x \notin \lambda_{p c} C l(\{y\})$. Thus $\lambda_{p c} C l(\{x\}) \neq \lambda_{p c} C l(\{y\})$. Hence by (4), $\lambda_{p c} C l(\{x\}) \cap \lambda_{p c} C l(\{y\})=\phi$, for each $y \in X \backslash U$. So $\lambda_{p c} C l(\{x\}) \cap\left[\cup\left\{\lambda_{p c} C l(\{y\}): y \in X \backslash U\right\}\right]=\phi$. Now, $U \in P O_{\lambda \mathrm{pc}}(X)$ and $y \in X \backslash U$, then $\{y\} \subseteq \lambda_{p c} C l(\{y\}) \subseteq \lambda_{p c} C l(X \backslash U)=X \backslash U$. Thus $X \backslash U=\bigcup\left\{\lambda_{p c} C l(\{y\})\right.$ : $y \in X \backslash U\}$. Hence, $\lambda_{p c} C l(\{x\}) \cap X \backslash U=\phi$, so $\lambda_{p c} C l(\{x\}) \subseteq U$. This implies that $(X, \tau)$ is $\lambda_{p c}-R_{0}$.

Theorem 4.21 Let $(X, \tau)$ be a topological space and $\lambda: P O(X) \rightarrow P(X)$ be any p-operation, then the following are equivalent:

1. $X$ is $\lambda_{p c}-T_{1}$.

2. $\lambda_{p c} C l(\{x\})=\{x\}$ for all $x \in X$.

3. $X$ is $\lambda_{p c}-R_{0}$ and $\lambda_{p c}-T_{0}$.

Proof. $(1) \Rightarrow(2)$ : Let $y \notin\{x\}$, then there exists $U \in P O_{\lambda p c}(X)$ such that $y \in U, x \notin U$, so $U \cap\{x\}=\phi$. Hence $y \notin \lambda_{p c} C l(\{x\})$ implies $\lambda_{p c} C l(\{x\}) \subseteq\{x\}$ also $\{x\} \subseteq \lambda_{p c} C l(\{x\})$ always, hence $\lambda_{p c} C l(\{x\})=\{x\}$ for all $x \in X$.

$(2) \Rightarrow(3):$ Let $x, y \in X$ with $x \neq y$. Then $\{x\}$ and $\{y\}$ are $\lambda_{p c}$-closed and hence $X \backslash\{x\}$ is a $\lambda_{p c}$-open set containing $y$ but not $x$. This shows that $X$ is $\lambda_{p c}-T_{0}$. Again, $x, y \in X$ with $x \neq y$, then $\lambda_{p c} C l(\{x\}) \neq \lambda_{p c} C l(\{y\})$. Also, $\lambda_{p c} C l(\{x\}) \cap \lambda_{p c} C l(\{y\})=\phi$. Thus, by Theorem 4.20, $X$ is $\lambda_{p c}-R_{0}$.

$(3) \Rightarrow(1):$ Let $x, y \in X$ with $x \neq y$. there exists $U \in P O_{\lambda p c}(X)$ such that $x \in U$ and $y \notin U$ then, $\lambda_{p c} C l(\{x\}) \subseteq U$ (as $X$ is $\left.\lambda_{p c}-R_{0}\right)$ and so $y \notin \lambda_{p c} C l(\{x\})$. Hence $x \in U \in P O_{\lambda p c}(X), y \notin U$ and $y \in X \backslash \lambda_{p c} C l(\{x\}) \in P O_{\lambda p c}(X)$, $x \notin X \backslash \lambda_{p c} C l(\{x\})$. Therefore, $X$ is a $\lambda_{p c}-T_{1}$ space.

Definition 4.22 Let $(X, \tau)$ be a topological space $\lambda: P O(X) \rightarrow P(X)$ be an p-operation. The space $X$ is said to be $\lambda_{p c}-R_{1}$ if for $x, y \in X$ with $\lambda_{p c} C l(\{x\}) \neq \lambda_{p c} C l(\{y\})$, there exist disjoint $\lambda_{p c}$-open sets $U$ and $V$ such that $\lambda_{p c} C l(\{x\}) \subseteq U$ and $\lambda_{p c} C l(\{y\}) \subseteq V$.

Theorem 4.23 If $\lambda: P O(X) \rightarrow P(X)$ is an p-operation and $X$ is $\lambda_{p c}-R_{1}$, then $X$ is $\lambda_{p c}-R_{0}$.

Proof. Let $U \in P O_{\lambda p c}(X)$ and $x \in U$. If $y \notin U$, then $\lambda_{p c} C l(\{x\}) \neq \lambda_{p c} C l(\{y\})$ ( as $\left.x \notin \lambda_{p c} C l(\{y\})\right)$. Hence there exists $V \in P O_{\lambda p c}(X)$ such that $\lambda_{p c} C l(\{y\}) \subseteq V$ and $x \notin V$. This gives that $y \notin \lambda_{p c} C l(\{x\})$, so $\lambda_{p c} C l(\{x\}) \subseteq U$. Hence, $X$ is a $\lambda_{p c}-R_{0}$ space.

By the following examples, we show the converse of above theorem is not true in general, and also we show $\lambda_{p c}-R_{0}$ and pre- $R_{0}$ are independent.

Example 4.24 Let $X=\{a, b\}$ and $\tau=\{\phi,\{a\}, X\}$. We define an p-operation $\lambda: P O(X) \rightarrow P(X)$ as $\lambda(A)=A$ if $A$ is empty and $\lambda(A)=X$ otherwise. Clearly $X$ is $\lambda_{p c}-R_{0}$, but it is neither pre- $R_{0}$ nor pre- $R_{1}$.

Example 4.25 Let $X=\{a, b\}$, and $\tau=P(X)$. We define an p-operation $\lambda: P O(X) \rightarrow P(X)$ as $\lambda(A)=A$ if $A$ is empty or $A=\{a\}$ and $\lambda(A)=X$ otherwise. Clearly $X$ is pre- $R_{0}$ and pre- $R_{1}$, but it is not $\lambda_{p c}-R_{0}$. 
Theorem 4.26 Let $(X, \tau)$ be a topological space $\lambda: P O(X) \rightarrow P(X)$ be an p-operation. Then the following are equivalent:

1. $X$ is $\lambda_{p c}-T_{2}$.

2. $X$ is $\lambda_{p c}-R_{1}$ and $\lambda_{p c}-T_{1}$.

3. $X$ is $\lambda_{p c}-R_{1}$ and $\lambda_{p c}-T_{0}$.

Proof. (1) $\Rightarrow(2)$ : Let $X$ be $\lambda_{p c}-T_{2}$, then $X$ is clearly $\lambda_{p c}-T_{1}$. Now if $x, y \in X$ with $\lambda_{p c} C l(\{x\}) \neq \lambda_{p c} C l(\{y\})$ then $x \neq y$, so there exist $U, V \in P O_{\lambda p c}(X)$ such that $x \in U, y \in V$ and $U \cap V=\phi$. Hence by Theorem 4.21, $\lambda_{p c} C l(\{x\})=\{x\} \subseteq U$ and $\lambda_{p c} C l(\{y\})=\{y\} \subseteq V$ and $U \cap V=\phi$. Therefore, $X$ is $\lambda_{p c}-R_{1}$.

$(2) \Rightarrow(3):$ It is obvious.

(3) $\Rightarrow(1)$ : Let $X$ be $\lambda_{p c}-R_{1}$ and $\lambda_{p c}-T_{0}$, then by Theorem 4.23, $X$ is $\lambda_{p c}-R_{0}$ and $\lambda_{p c}-T_{0}$. Hence, by Theorem 4.21, $X$ is $\lambda_{p c}-T_{1}$. If $x, y \in X$ with $x \neq y$, then $\lambda_{p c} C l(\{x\})=\{x\} \neq\{y\}=\lambda_{p c} C l(\{y\})$. Since $X$ is $\lambda_{p c}-R_{1}$, so there exist $U, V \in P O_{\lambda p c}(X)$ such that $\lambda_{p c} C l(\{x\})=\{x\} \subseteq U, \lambda_{p c} C l(\{y\})=\{y\} \subseteq V$ and $U \cap V=\phi$. Hence, $X$ is $\lambda_{p c}-T_{2}$.

\section{References}

[1] B. Ahmad and S. Hussain, Properties of $\gamma$-operations in topological spaces, Aligarh Bull. Math., 22(1):45-51, 2003.

[2] H. Maki, J. Umehara, and T. Nori, Every Topological Space is Pre-T ${ }_{1 / 2}$, Mem. Fac. Sci. Kochi Univ. Ser. A(Mathes.), 17(1996), 33-42.

[3] S. G. Crossley and S. K. Hildebrand, Semi-closure, Texas J. Sci., 22:99-112, 1971.

[4] H. Corson and E. Michael, Metrizability of certain countable union, Illinois J. Math., 8(1964), 351-360.

[5] M. Caldas, S. Jafari, and T. Nori, Characterizations of pre- $R_{0}$, and pre- $R_{1}$ topological spaces, Topology Proceedings, $25(2000), 17-30$.

[6] S. Kasahara, Operation-compact spaces, Math. Japonica, 24:97-105, 1979.

[7] A. S. Mashhour, M. E. Abd El-Monsef and S. N. El-Deeb, On pre-continuous and week precontinuous mappings, Proc. Math. Phys. Soc., [Egypt], 53(1982), 47-53.

[8] N. Levine, Generalized closed sets in topology, Rend. Circ. Math. Palermo, 19:89-96, 1970.

[9] A. Chattopadhyay, pre- $T_{0}$ and pre- $T_{1}$ Topological Spaces, J. Indian Acad. Math., 17(2),(1995), $156-159$.

[10] H. Ogata, Operation on topological spaces and associated topology, Math. Japonica, 36(1):175 -184, 1991. 